\title{
Expansion of Dawson's Function in a Series of Chebyshev Polynomials
}

\author{
By David G. Hummer
}

\begin{abstract}
Dawson's function
\end{abstract}

$$
F(x)=e^{-x^{2}} \int_{0}^{x} e^{t^{2}} d t=\int_{0}^{\infty} e^{-t^{2}} \sin 2 x t d t
$$

is of importance, for instance, in the calculation of profiles of absorption lines [1], [2]. Extensive tables of $F(x)$ are given by Miller \& Gordon [3], Rosser [4], and Lomander \& Rittsten [5]; the last of these is the most satisfactory. Terrill \& Sweeny [6] tabulate $e^{x^{2}} F(x)$. For use in machine computing in some astrophysical problems in which severe cancellation occurs, we have obtained a Chebyshev expansion of $F(x)$ capable of very high accuracy in the interval $-k \leqq x \leqq k$, where $k$ is sufficiently large so that, for $x>k, F(x)$ may be obtained from the asymptotic series

$$
F(x) \sim \frac{1}{2 x}+\frac{1}{2^{2} x^{3}}+\frac{1 \cdot 3}{2^{3} x^{5}}+\frac{1 \cdot 3 \cdot 5}{2^{4} x^{7}}+\cdots
$$

Since $F(x)$ is an odd function, we write

$$
F(k x)=\sum_{n=0}^{\infty} a_{n}(k) T_{2 n+1}(x), \quad-1 \leqq x \leqq 1
$$

where

$$
T_{m}(x)=\cos \left(m \cos ^{-1} x\right) .
$$

From the orthogonality of the $T_{m}(x)$ we have

$$
a_{n}(k)=\frac{2}{\pi} \int_{0}^{\pi} F(k \cos \theta) \cos (2 n+1) \theta d \theta
$$

Integrating by parts and using the differential equation

$$
F^{\prime}(x)=1-2 x F(x),
$$

we have

$$
\begin{aligned}
a_{n}(k) & =\frac{2}{\pi} \frac{k}{2 n+1} \int_{0}^{\pi}[1-2 k \cos \theta F(k \cos \theta)] \sin \theta \sin (2 n+1) \theta d \theta \\
& =\frac{1}{\pi} \frac{k^{2}}{2 n+1} \int_{0}^{\pi} F(k \cos \theta)[\cos (2 n+3) \theta-\cos (2 n-1) \theta] d \theta
\end{aligned}
$$

or

$$
a_{n}(k)=\frac{k^{2}}{2(2 n+1)}\left[a_{n+1}(k)-a_{n-1}(k)\right] .
$$

The coefficients $a_{n}$ may be obtained by the well-known method (see for example Received May 27, 1963. 
[7], p. 88-90) of setting

$$
\tilde{a}_{N}=1, \quad \tilde{a}_{N+1}=0
$$

and obtaining $\tilde{a}_{N-1}, \cdots, \tilde{a}_{0}$ recursively from (4). Then

$$
F^{*}(k x)=c \sum_{n=0}^{N} \tilde{a}_{n}(k) T_{2 n+1}(x)
$$

and $c$ is obtained from the condition $\frac{d}{d x} F(0)=1$,

$$
c=k / \sum_{n=0}^{N}(-1)^{n}(2 n+1) \tilde{a}_{n}(k) .
$$

The coefficients ${a_{n}}^{*}(k)=c \tilde{a}_{n}$ have been evaluated with $N=35$ using doubleprecision arithmetic on the University of London Mercury Computer. In Table 1 we give $a_{0}{ }^{*}, \cdots, a_{33}^{*}$ for $k=5.0$. The values of $F(x)$ obtained by summing thirty terms in the series using the summation algorithm of Clenshaw [8] agree with the twenty-place value of Lomander and Rittsten to within two places in the 14th place. By including the terms corresponding to $n=30, \cdots 33$, the error should be reduced to a few units in the 15 th place.

The coefficients $a_{n}(k)$ may also be evaluated analytically. Substituting the second form of $F(x)$ given in (1) into (3) and interchanging the order of integration, we have

\begin{tabular}{|c|c|c|c|}
\hline$n$ & $a_{n}^{*}(5)$ & $n$ & $a_{n}^{*}(5)$ \\
\hline 0 & .1999999999972224 & 17 & -.0000027876379719 \\
\hline 1 & -.1840000000029998 & 18 & .0000008566873627 \\
\hline 2 & .1558399999965025 & 19 & -.0000002518433784 \\
\hline 3 & -.1216640000043988 & 20 & .0000000709360221 \\
\hline 4 & .0877081599940391 & 21 & -.0000000191732257 \\
\hline 5 & -.0585141248086907 & 22 & .0000000049801256 \\
\hline 6 & $.03621573 \quad 01623914$ & 23 & $-.00000000 \quad 12447734$ \\
\hline 7 & -.0208497654398036 & 24 & .0000000002997777 \\
\hline 8 & $.01119601 \quad 16346270$ & 25 & -.0000000000696450 \\
\hline 9 & -.0056231896167109 & 26 & .0000000000156262 \\
\hline 10 & $.00264876 \quad 34172265$ & 27 & -.0000000000033897 \\
\hline 11 & -.0011732670757704 & 28 & .0000000000007116 \\
\hline 12 & $.00048995 \quad 19978088$ & 29 & -.0000000000001447 \\
\hline 13 & -.0001933630801528 & 30 & .0000000000000285 \\
\hline 14 & .0000722877446788 & 31 & -.0000000000000055 \\
\hline 15 & $-.00002565 \quad 55124979$ & 32 & .0000000000000010 \\
\hline 16 & .0000086620736841 & 33 & -.0000000000000002 \\
\hline
\end{tabular}

$$
a_{n}(k)=\frac{2}{\pi} \int_{0}^{\infty} e^{-t^{2}} \int_{0}^{\pi} \sin (2 k \cos \theta) \cos (2 n+1) \theta d \theta
$$

Using some standard results from the theory of Bessel functions, we transform 
this to

$$
\begin{aligned}
a_{n}(k) & =(-1)^{n} 2 \int_{0}^{\infty} e^{-t^{2}} J_{2 n+1}(2 k t) d t \\
& =(-1)^{n} \sqrt{\pi} e^{-k^{2} / 2} I_{n+(1 / 2)}\left(k^{2} / 2\right) \\
& =\sum_{r=0}^{n} \frac{(n+r) !}{r !(n-r) !} k^{-2 r-1}\left[(-1)^{r+n}-e^{-k^{2}}\right], \quad n=0,1,2 \cdots .
\end{aligned}
$$

This expression may easily be seen to be consistent with (4).

Acknowledgments. This work was supported by the Office of Naval Research.

Department of Physics,

University College London,

Gower Street,

London, W. C. 1.

1. D. Harris III, "On the line-absorption coefficient due to Doppler effect and damping," Astrophys. J., v. 108, 1948, p. 112-115.

2. D. G. Hummer, "Noncoherent scattering. I. The redistribution functions with Doppler broadening," Monthly Notices Roy. Astronom. Soc., v. 125, 1963, p. 21-37.

3. W. L. Mrller \& A. R. Gordon, "Numerical evaluation of infinite series and integrals which arise in certain problems of linear heat flow, electrochemical diffusion, etc.," J. Chem. Phys., v. 35, 1935, p. 2785-2884.

4. J. B. Rossen, Theory and Application of $\int_{0}^{\infty} e^{-x^{2}} d x$ and $\int_{0}^{z} e^{-p^{2} y^{2}} d y \int_{0}^{y} e^{-x^{2}} d x$, Mapleton House, Brooklyn, N. Y., 1948.

5. B. Lohmander \& S. Rittsten, "Tables of the function $y=e^{-x^{2}} \int_{0}^{x} e^{t^{2}} d t$," Kungl. Fysiogr. Sällsk. $i$ Lund Förh., v. 28, 1958, p. 45-52.

6. H. M. Terrill \& L. Sweeny, "An extension of Dawson's table of the integral of $e^{x^{2}}$," J. Franklin Inst., v. 237, 1944, p. 495-497; "Table of the integral of $e^{x^{2}}$," ibid., v. 238, 1944, p. $220-222$.

7. National Physical Laboratory, Modern Computing Methods, 2nd edition, H. M. Stationery Office, London, 1961.

8. C. W. Clenshaw, "A note on the summation of Chebyshev series," $M T A C$, v. 9, 1955, p. 118-120; see also [7], Chapter 8.

\section{First One Hundred Zeros of $J_{0}(x)$ Accurate to 19 Significant Figures}

\section{By Henry Gerber}

1. Introduction. Some physical investigations require a knowledge of accurate values of the zeros of the Bessel function $J_{0}(x)$. The most extensive values previously published are those of the British Association for the Advancement of Science [1], which consist of 10 decimal places. More accurate values have now been computed, and are presented in Table 1. The minimum accuracy of the tabulated zeros is 19 significant figures.

2. Method of Computation. Two methods were used to compute the roots. The first twelve roots were computed by the method of "false position." The values of

Received August 12, 1963. 